\title{
Exome sequencing of multiple-sclerosis patients and their unaffected first-degree relatives
}

\author{
Sheila Garcia-Rosa' ${ }^{1}$ Maria Galli de Amorim', Renan Valieris², Vanessa Daccach Marques ${ }^{3,4}$, \\ Julio Cesar Cetrulo Lorenzi ${ }^{4,5}$, Vania Balardin Toller ${ }^{6}$, Guilherme Sciascia do Olival ${ }^{6}$, \\ Wilson Araújo da Silva Júnior ${ }^{4,5}$, Israel Tojal da Silva ${ }^{2}$, Amilton Antunes Barreira ${ }^{3,4}$, Diana Noronha Nunes ${ }^{1}$ \\ and Emmanuel Dias-Neto ${ }^{1,7^{*}}$ (D)
}

\begin{abstract}
Objectives: The understanding of complex multifactorial diseases requires the availability of a variety of data for a large-number of affected individuals. In this data note here we provide whole exome sequencing data from a set of non-familiar multiple-sclerosis (MS) patients as well as their unaffected first-degree relatives. This data might help the identification of genomic alterations, including single nucleotide polymorphisms, de novo variations and structural genomic variations, such as copy-number alterations that may impact this disease.

Data description: This dataset comprises the full exome of 28 Brazilian subjects grouped in eight distinct families, consisting of four complete trios (mother-patient-father) plus another four complete trios with one added unaffected sibling. In total, we present the full exome data of eight patients diagnosed with recurrent remittent multiple sclerosis. Diagnoses were made by experienced neurologists and all enrolled patients had at least 5 years of follow up and specific MS treatment. Exomes were sequenced from leukocyte-derived DNA, after the capture of exons using biotinylated probes, in the lon Proton platform. For each exome we generated an average of 66.1 million good quality mapped reads with an average length of $\sim 160$ nt. On average, for $90 \%$ of the exome a vertical coverage above $20 x$ was reached.
\end{abstract}

Keywords: Whole exome sequencing, Inheritance, 'de novo', Remittent-recurrent, Multiple sclerosis

\section{Objective}

The understanding of complex multifactorial diseases requires the availability of information for thousands of affected individuals. The release of this data is an attempt to contribute to studies of remittent recurrent multiple-sclerosis (RRMS). The sequencing data presented here was generated to help the identification of genomic variants located in the exomes of RRMS patients from Brazil, a country with elevated ethnic admixture [1-3]. Exomes of the patient's first-degree

\footnotetext{
*Correspondence: emmanuel@cipe.accamargo.org.br

1 Lab. of Medical Genomics, International Research Center, A.C.Camargo Cancer Center, Rua Taguá 440, 1st Floor, São Paulo, SP 01508-010, Brazil

Full list of author information is available at the end of the article
}

unaffected relatives (both parents and siblings) were also sequenced to allow the study of 'de novo' variations which, so far, have been under investigated in RRMS. The exome variations observed here may help the study of relevant single nucleotide polymorphisms, de novoand structural genomic variations-such as copy-number alterations that may be associated with the disease [4-8]. In total, 28 individuals have been sequenced including eight RRMS patients as well as a set of another 20 unaffected first-degree relatives. Some variations suggested by these data have been validated by Sanger sequencing and subjected to functional analysis, have been presented in a manuscript that is currently under review (Garcia-Rosa, et al. unpublished). Most of the exomes presented here have not been explored 
yet, but it is certain that the whole dataset can be useful for subsequent studies by other groups interested in this field.

\section{Data description}

This study was focused on families containing one member diagnosed with RRMS, with both biological parents alive, and no description of other cases of neurodegenerative or neuropsychiatric cases in the family. Subjects were diagnosed as RRMS patients by experienced neurologists, using currently accepted protocols [9]; paternity and maternity were confirmed for all families using the Variant Call Format (VCFs) files to verify the compatibility status of single nucleotide polymorphisms for all patients and their siblings, according to their status in the exomes of the parents [10]. The study comprised a total of 28 Brazilian individuals, divided in eight families. The trio mother-patient-father was available for all eight families and four of the families also included one unaffected sibling. According to this, subjects were designated in familial groups (G) from G1 to G8. For each family group (G), individuals were classified with single letters designating the mother $(\mathrm{M})$, the father $(\mathrm{F})$ and the patient $(\mathrm{P})$, as well as the unaffected patient-brother (B) or the unaffected patientsister (S). Therefore, G1F indicates the exome sequence of the father of group 1 and G5S indicates the exome sequence of the unaffected sister of the patient of family 5 , and so forth. For each subject, genomic DNA was obtained from leukocytes isolated from $4 \mathrm{~mL}$ samples of peripheral whole blood using the Wizard Genomic DNA purification kit (Promega, USA). One microgram of genomic DNA samples from eight affected individuals (G1P to G8P), their parents (16 samples: G1 M to G8 $\mathrm{M}$ for mothers and G1F to G8F for fathers) as well as four siblings (G2B, G4S, G5S and G6S) were prepared for WES (whole exome sequencing) using the Ion TargetSeq Exome Capture Kit (Thermo Fisher, USA). For this, the DNA was fragmented for $30 \mathrm{~min}$ (Ion Shear Plus Enzyme Mix II), adapters and barcodes were ligated and size selected (275-295 bp). Libraries were amplified (8 cycles) using the Platinum PCR Super Mix High Fidelity. Exons were captured from 500 ng of amplified libraries by hybridization using biotinylated probes (Ion-TargetSeq-Exome-50 Mb-hg19_RevA), following the manufacturer's instructions. This panel covers a total of 46.2 million bases, encompassing 25,313 genes and 267,049 targets. The instructions to access and to download the corresponding target regions (.bed files) of this specific panel are provided by the manufacturer [11].

WES libraries were sequenced using the Ion PI Sequencing 200 kit V3 in the Ion Proton sequencing platform (CIPE, A.C.Camargo Cancer Center, São Paulo, SP, Brazil). On average, each sequencing run in a P1 chip generated about 10-12 billion mapped bases. Sequencing reads were mapped against the specific exome target region (Ion-TargetSeqExome-50 Mb-hg19_revA), using the configuration TargetSeq-Proton-Germ Line-High Stringency, and the Torrent Suite V4.2.1. For each individual exome we generated an average of 66.1 million good quality mapped reads with an average length of $\sim 160 \mathrm{nt}$. On average, for $90 \%$ of the exome we reached a vertical coverage of at least $20 \times$. The data described in the present report has not been filtered prior to deposition in the short reads archive (/sra) of NCBI, therefore, mapped and unmapped reads are available through the provided links shown in Table 1.

\section{Limitations}

The sequencing platform used here generates high quality reads, but homopolymeric regions-the consecutive repetition of the same nucleotide for five times or more-have been shown to contain higher error rates that affect the length of the repetitive unit [12]. Previous work recommended that variants located in the junction of two homopolymers, as well as variants that indicate the elongation (insertions) or the shortening (deletions) of homopolymeric repeats, should be confirmed by Sanger sequencing [12]. Therefore, variants in homopolymeric regions identified in our data should be evaluated with extra caution. Compared to other Next Generation Sequencing platforms, such as Illumina, the platform used here presents higher error rates in variants identified as small insertions and deletions (InDels). Also, as the number of individuals sequenced here is small, investigators should seek more data and experimental validation to help identifying genomic alterations that might be associated with RRMS. 
Table 1 Overview of data files/data sets

\begin{tabular}{|c|c|c|c|c|}
\hline Label & Name of data file & File types (file extension) & $\begin{array}{l}\text { Data repository and identifier } \\
\text { (DOI or accession number) }\end{array}$ & License \\
\hline Data file 1 & SAMN07947504-G1M & \multirow{28}{*}{$\begin{array}{l}\text { Binary sequence } \\
\text { alignment/map } \\
\text { file (.bam) }\end{array}$} & \multirow{28}{*}{$\begin{array}{l}\text { Sequence Read Archive } \\
\text { http://www.ncbi.nlm.nih.gov/sra } \\
\text { (SRP122913) }\end{array}$} & \multirow[t]{28}{*}{ CC-BY } \\
\hline Data file 2 & SAMN07947505-G1P & & & \\
\hline Data file 3 & SAMN07947506-G1F & & & \\
\hline Data file 4 & SAMN07947507-G2B & & & \\
\hline Data file 5 & SAMN07947508-G2M & & & \\
\hline Data file 6 & SAMN07947509-G2P & & & \\
\hline Data file 7 & SAMN07947510-G2F & & & \\
\hline Data file 8 & SAMN07947511-G3M & & & \\
\hline Data file 9 & SAMN07947512-G3P & & & \\
\hline Data file 10 & SAMN07947513-G3F & & & \\
\hline Data file 11 & SAMN07947514-G4S & & & \\
\hline Data file 12 & SAMN07947515-G4M & & & \\
\hline Data file 13 & SAMN07947516-G4P & & & \\
\hline Data file 14 & SAMN07947517-G4F & & & \\
\hline Data file 15 & SAMN07947518-G5S & & & \\
\hline Data file 16 & SAMN07947519-G5M & & & \\
\hline Data file 17 & SAMN07947520-G5P & & & \\
\hline Data file 18 & SAMN07947521-G5F & & & \\
\hline Data file 19 & SAMN07947522-G6S & & & \\
\hline Data file 20 & SAMN07947523-G6M & & & \\
\hline Data file 21 & SAMN07947524-G6P & & & \\
\hline Data file 22 & SAMN07947525-G6F & & & \\
\hline Data file 23 & SAMN07947526-G7M & & & \\
\hline Data file 24 & SAMN07947527-G7P & & & \\
\hline Data file 25 & SAMN07947528-G7F & & & \\
\hline Data file 26 & SAMN07947529-G8M & & & \\
\hline Data file 27 & SAMN07947530-G8P & & & \\
\hline Data file 28 & SAMN07947531-G8F & & & \\
\hline
\end{tabular}

\section{Abbreviations}

RRMS: remittent recurrent multiple sclerosis; WES: whole exome sequencing; VCF: variant call format; InDels: insertions and deletions (InDels).

\section{Authors' contributions}

Genomics planning, data analysis and project management: SGR, MGA, DNN EDN; Diagnosis, patient selection/recruitment and clinical evaluation: JCCL, WAS, AAB, VDM, GSO, VBT; Bioinformatics: RV, ITS; Manuscript writing and overall project coordination: EDN. All authors have read and agreed with the submission of this manuscript. All authors read and approved the final manuscript.

\footnotetext{
Author details

${ }^{1}$ Lab. of Medical Genomics, International Research Center, A.C.Camargo Cancer Center, Rua Taquá 440, 1st Floor, São Paulo, SP 01508-010, Brazil. ${ }^{2}$ Laboratory of Computational Biology and Bioinformatics, International Research Center, A.C.Camargo Cancer Center, Rua Taguá 440, 1st Floor, São Paulo, SP 01508-010, Brazil. ${ }^{3}$ Department of Neurosciences, Clinical Neuroimmunology Division, Medical School and Hospital das Clínicas of Ribeirão Preto, University of São Paulo (USP), Avenida Bandeirantes, 3900, Ribeirão Preto, SP 14049-900, Brazil. ${ }^{4}$ Center for Medical Genomics, HCFMRP/USP, Avenida Bandeirantes, 3900, Ribeirão Preto, SP 14049-900, Brazil. ${ }^{5}$ Department of Genetics, Ribeirão Preto Medical School, University of São Paulo (USP), Avenida Bandeirantes, 3900, Ribeirão Preto, SP 14049-900, Brazil. ${ }^{6}$ Neurosciences Research Group,
}

Faculdade de Ciências Médicas da Santa Casa de São Paulo, Rua Doutor Cesário Motta Júnior, 61 - Vila Buarque, São Paulo, SP 01221-020, Brazil. ${ }^{7}$ Lab. of Neurosciences (LIM-27), Institute of Psychiatry, Faculdade de Medicina, Universidade de São Paulo, São Paulo, SP, Brazil.

\section{Acknowledgements}

The authors the support given by the A.C.Camargo Cancer Center.

\section{Competing interests}

The authors declare that they have no competing interests.

\section{Availability of data}

The dataset generated during the current study is available in the http://www ncbi.nlm.nih.gov/sra (Accession Number: SRP122913). Please see Table 1 for details.

\section{Consent for publication}

Not applicable.

\section{Ethics approval and consent to participate}

All patients agreed to participate in this study and signed an informed consent form. The study was approved by the "Comitê de Ética em Pesquisa" of the A.C.Camargo Cancer Center (protocol 1748/13) and this approval was recognized and accepted by the "Comitê de Ética em Pesquisa em Seres 
Humanos, Hospital das Clínicas da Faculdade de Medicina de Ribeirão Preto da Universidade de São Paulo (CEP, HCRP, FMUSPRP)", in 12/04/2013.

\section{Funding}

Mr. Waldemar Benassi, Associação Beneficente Alzira Denise Hertzog Silva (ABADHS), Coordenação de Aperfeiçoamento de Pessoal de Nível Superior (CAPES, Brazil), Conselho Nacional de Desenvolvimento Científico e Tecnológico (CNPq, Brazil - Grant: 480138/2013-3) and Fundação de Amparo à Pesquisa do Estado de São Paulo (Grants: 2013/24293-7). WASJr, AAB and ED-N are research fellows from CNPq, Brazil.

\section{Publisher's Note}

Springer Nature remains neutral with regard to jurisdictional claims in published maps and institutional affiliations.

Received: 4 November 2017 Accepted: 6 December 2017 Published online: 12 December 2017

\section{References}

1. Homburger JR, Moreno-Estrada A, Gignoux CR, Nelson D, Sanchez E, Ortiz-Tello P, et al. Genomic insights into the ancestry and demographic history of South America. PLoS Genet. 2015;11:e1005602.

2. Kehdy FSG, Gouveia MH, Machado M, Magalhães WCS, Horimoto AR, Horta BL, et al. Origin and dynamics of admixture in Brazilians and its effect on the pattern of deleterious mutations. Proc Natl Acad Sci USA. 2015;112:8696-701
3. Parra FC, Amado RC, Lambertucci JR, Rocha J, Antunes CM, Pena SDJ. Color and genomic ancestry in Brazilians. Proc Natl Acad Sci USA. 2003;100:177-82

4. Kong J, Shin J, Won J, Lee K, Lee U, Yoon J. ExCNVSS: A noise-robust method for copy number variation detection in whole exome sequencing data. Biomed Res Int. 2017;2017:9631282.

5. Tan R, Wang J, Wu X, Juan L, Zheng L, Ma R, et al. ERDS-exome: a hybrid approach for copy number variant detection from whole-exome sequencing data. IEEE/ACM Trans Comput Biol Bioinforma. 2017:5963:11. http://ieeexplore.ieee.org/document/8057779/. Accessed 10 Dec 2017.

6. DePristo MA, Banks E, Poplin R, Garimella KV, Maguire JR, Hartl C, et al. A framework for variation discovery and genotyping using next-generation DNA sequencing data. Nat Genet. 2011;43:491-8.

7. Rimmer A, Phan H, Mathieson I, lqbal Z, Twigg SRF, Wilkie AOM, et al. Integrating mapping-, assembly- and haplotype-based approaches for calling variants in clinical sequencing applications. Nat Genet. 2014;46:912-8.

8. Liu Y, Li B, Tan R, Zhu X, Wang Y. A gradient-boosting approach for filtering de novo mutations in parent-offspring trios. Bioinformatics. 2014;30:1830-6.

9. Polman $\mathrm{CH}$, Reingold SC, Banwell B, Clanet M, Cohen JA, Filippi M, et al. Diagnostic criteria for multiple sclerosis: 2010 revisions to the McDonald criteria. J Neurol. 2014;26:490-9.

10. Ryan A, Baner J, Demko Z, Hill M, Sigurjonsson S, Baird ML, Rabinowitz M. Informatics-based, highly accurate, non-invasive prenatal paternity testing. Genet Med. 2013;15:473-7.

11. https://assets.thermofisher.com/TFSAssets/LSG/manuals/MAN0006730_ TargetSegExomeEnrich IonProton_UG.pdf. Accessed 5 Dec 2017.

12. Fujita S, Masago K, Okuda C, Hata A, Kaji R, Katakami N, Hirata Y. Single nucleotide variant sequencing errors in whole exome sequencing using the ion proton system. Biomed Rep. 2017;7:17-20.

\section{Submit your next manuscript to BioMed Central and we will help you at every step:}

- We accept pre-submission inquiries

- Our selector tool helps you to find the most relevant journal

- We provide round the clock customer support

- Convenient online submission

- Thorough peer review

- Inclusion in PubMed and all major indexing services

- Maximum visibility for your research

Submit your manuscript at www.biomedcentral com/submit
O Biomed Central 